\title{
Porphyromonas gingivalis exacerbates the progression of fatty liver disease via CD36-PPAR $\gamma$ pathway
}

\author{
Ji-Su Ahn ${ }^{1,2, \#}$, Ji Won Yang ${ }^{1,2, \#}$, Su-Jeong O ${ }^{1,2}$, Ye Young Shin ${ }^{1,2}$, Min-Jung Kang ${ }^{3}$, Hae Ryoun Park ${ }^{3,4}$, \\ Yoojin Seo ${ }^{3, *}$ E Hyung-Sik Kim ${ }^{1,2,3, *}$ \\ ${ }^{1}$ Department of Life Science in Dentistry, School of Dentistry, Pusan National University, Yangsan 50612, ${ }^{2}$ Department of Oral \\ Biochemistry, School of Dentistry, Pusan National University, Yangsan 50612, ${ }^{3}$ Periodontal Disease Signaling Network Research Center \\ Dental \& Life Science Institute, Pusan National University, Yangsan 50612, ${ }^{4}$ Department of Oral Pathology, School of Dentistry, Pusan \\ National University, Yangsan 50612, Korea
}

\begin{abstract}
Periodontal diseases have been reported to have a multidirectional association with metabolic disorders. We sought to investigate the correlation between periodontitis and diabetes or fatty liver disease using HFD-fed obese mice inoculated with $\boldsymbol{P}$. gingivalis. Body weight, alveolar bone loss, serological biochemistry, and glucose level were determined to evaluate the pathophysiology of periodontitis and diabetes. For the evaluation of fatty liver disease, hepatic nonalcoholic steatohepatitis (NASH) was assessed by scoring steatosis, inflammation, hepatocyte ballooning and the crucial signaling pathways involved in liver metabolism were analyzed. The C-reactive protein (CRP) level and NASH score in $P$. gingivalis-infected obese mice were significantly elevated. Particularly, the extensive lobular inflammation was observed in the liver of obese mice infected with $P$. gingivalis. Moreover, the expression of metabolic regulatory factors, including peroxisome proliferator-activated receptor $\gamma($ Ppary) and the fatty acid transporter Cd36, was up-regulated in the liver of $\boldsymbol{P}$. gingivalis-infected obese mice. However, inoculation of $\boldsymbol{P}$. gingivalis had no significant influence on glucose homeostasis, insulin resistance, and hepatic mTOR/AMPK signaling. In conclusion, our results indicate that $\boldsymbol{P}$. gingivalis can induce the progression of fatty liver disease in HFD-fed mice through the upregulation of CD36-PPAR $\gamma$ axis. [BMB Reports 2021; 54(6): 323-328]
\end{abstract}

\footnotetext{
*Corresponding authors. Hyung-Sik Kim, Tel: +82-51-510-8231; Fax: +82-51-510-8210; E-mail: hskimcell@pusan.ac.kr; Yoojin Seo, Tel: +82-51-510-0311; Fax: +82-51-510-8210; E-mail: amaicat24@ naver.com

${ }^{\#}$ These authors contributed equally to this work.
}

https://doi.org/10.5483/BMBRep.2021.54.6.050

Received 8 April 2021, Revised 21 April 2021, Accepted 28 April 2021

Keywords: Diabetes, Inflammation, Lipid metabolic disorder, Nonalcoholic fatty liver disease, Periodontitis, Porphyromonas gingivalis

\section{INTRODUCTION}

Periodontitis is a chronic dental disease with a high risk of recurrence, in which the periodontal tissue is destroyed by bacteria accumulating in the teeth (1). Several risk factors such as poor oral health, hormonal changes, bacterial infection have been reported to be associated with the initiation and progression of periodontal disease (2). Periodontitis is mostly caused by gram-negative bacteria such as Aggregatibacter actinomycetemcomitans, Porphyromonas gingivalis, Prevotella intermedia, and Bacteroides forsythus (3). Recent reports have suggested the association of periodontitis with systemic diseases including cardiovascular disease, rheumatoid arthritis, type 2 diabetes mellitus (T2D), hypertension and inflammatory bowel disease (4-6).

Diabetes is accompanied by complications such as hypertension, cardiovascular disease, skin lesions, and obesity $(7,8)$. A multidirectional relationship between diabetes and periodontitis has been reported, with diabetes increasing the risk of periodontitis and periodontal inflammation negatively affecting control of blood glucose levels (9-11). Non-alcoholic fatty liver disease (NAFLD) is frequently observed in patients with T2D and is known to be associated with increased insulin resistance triggered by fatty acid accumulation, inflammation and reactive oxygen species production (12-14). Moreover, it has been reported in many studies that periodontitis contributes to the progression of NAFLD $(15,16)$.

It has been shown that hepatic CD36, which acts as a transcriptional regulator of PPAR $\gamma$, is significantly upregulated in obese rats with periodontitis (17). Depletion of CD36 from hepatocytes attenuates fatty liver disease and ameliorates insulin sensitivity in obese mice (18). As another important fatty acid regulator, the fatty acid-binding protein 4 (FABP4) can induce systemic disease progression (19). FABP4 expression is increased in macrophages upon infection with $P$. gingivalis or Fusobacterium nucleatum, affecting both $\mathrm{T} 2 \mathrm{D}$ and periodontitis progression (20). In addition, adipocytes from patients with T2D have been reported to contain reduced levels of FOXO1, an important regulator of glucose and lipid homeostasis (21).

ISSN: 1976-670X (electronic edition)

Copyright (C) 2021 by the The Korean Society for Biochemistry and Molecular Biology

c) This is an open-access article distributed under the terms of the Creative Commons Attribution Non-Commercial License (http://creativecommons.org/licenses/by-nc/4.0) which permits unrestricted non-commercial use, distribution, and reproduction in any medium, provided the original work is properly cited. 
The link between periodontitis and T2D or fatty liver disease is associated with the production and expression of inflammatory factors including pro-inflammatory cytokines, chemokines, and metabolic regulators (22). The higher levels of tumor necrosis factor $\alpha$ (TNF $\alpha$ ), interleukin (IL)-1 $\beta$, and CRP induce insulin resistance by impairing insulin action $(23,24)$. A marked elevation of components of the NLR Family Pyrin Domain Containing 3 (NLRP3) inflammasome at both the gene and protein levels have been reported in patients with periodontitis and T2D (25). Moreover, several inflammatory responses mediated by pro-inflammatory cytokines or inflammasome activation have been suggested to play an important role in the induction of hepatic inflammation in NASH $(26,27)$.

Since obesity is a major cause for chronic diseases including T2D and NAFLD (28), we used HFD-induced obese mice model. To investigate the correlation of periodontitis and T2D or fatty liver disease, HFD-fed mice were inoculated with $P$. gingivalis to develop the periodontitis, and then checked for crucial pathophysiological factors related to the link between the diseases.

\section{RESULTS}

Obese mice with $\boldsymbol{P}$. gingivalis infection exhibit alveolar bone loss and increased CRP level

To investigate the correlation between periodontitis and liver malfunction, periodontitis was induced by $P$. gingivalis infection on the mandibular molars of 8-week old mice or by direct administration into the stomach using a feeding catheter. HFD was supplied to the mice to generate a mouse model of obesity (Fig. 1A). Body weight was measured once every 2 weeks during the experiment. $P$. gingivalis treatment increased the body weight of mice. Although the body weight of HFD-fed groups significantly increased compared to the NC group, there was no difference among HFD-fed (HFD), HFDfed P. gingivalis-infected (HFD-p.g.), and HFD-p.g. (P.O.) groups (Fig. 1B). To confirm that the experimental periodontitis was appropriately induced by $P$. gingivalis infection, the extent of alveolar bone loss was measured. The mice infected with $P$. gingivalis in the oral cavity displayed a significant increase in alveolar bone loss compared to the NC group, as indicated by a decrease in the relative amounts of bone around the mandibular molars of the infected mice (Fig. 1C). We next performed serum biochemical analysis by measuring glucose, metabolic disease-related factors (cholesterol, HDL, LDL), liver enzymes (AST, ALT), and the levels of the systemic inflammatory marker (CRP) in blood. The levels of glucose, cholesterol, $\mathrm{HDL}, \mathrm{LDL}, \mathrm{AST}$ and ALT were significantly increased in HFDfed mice. However, $P$. gingivalis treatment did not further elevate the levels of glucose, cholesterol and lipoproteins, as well as liver enzymes. CRP levels were significantly increased in $P$. gingivalis-treated groups compared to those in the NC group (Fig. 1D). These results indicate that HFD feeding can induce systemic metabolic disease with liver damage and that the $P$. gingivalis infection does not alter the overall levels of serum biochemical factors except the CRP level.

\section{$P$. gingivalis infection did not affect the glucose metabolism in HFD-fed mice}

We next measured blood glucose using intraperitoneal glucose tolerance test (IPGTT) to investigate the bidirectional relationship between periodontitis and glucose homeostasis. After 6 hours of fasting, the blood glucose levels were measured. Fast-
A

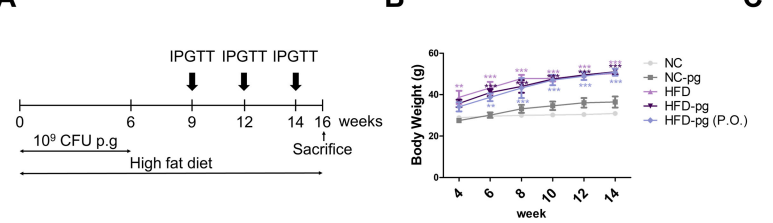

D

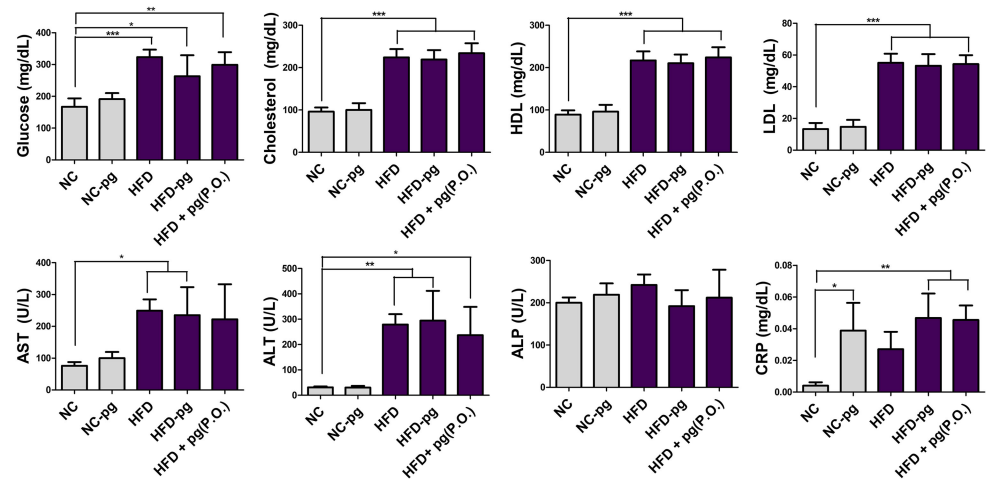

C

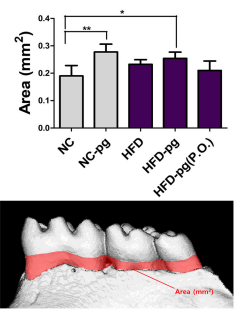
serological biochemical analysis in blood samples. (A) Mice were divided into 5 groups: NC, NC-p.g., HFD, HFD-p.g., and HFD-p.g.(P.O.). Mice were maintained on HFD for 16 weeks, and periodontitis was induced by $P$. gingivalis infection of the mandibular first molars or by direct administration into the stomach using a feeding needle catheter 3 times a week. (B) Measurement of body weight every 2 weeks. (C) Hemi-mandible, as reconstructed by micro-computed tomography (micro-CT) analysis, and alveolar bone loss (red area) were measured for each group. (D) Levels of glucose, cholesterol, HDL, LDL, AST, ALT, ALP, and CRP in the blood from mice of each group at 14 weeks. The results are shown as the mean $\pm \mathrm{SD}$. $* \mathrm{P}<$ $0.05,{ }^{* * P}<0.01,{ }^{* * *} \mathrm{P}^{ \pm}<0.001$. 
A

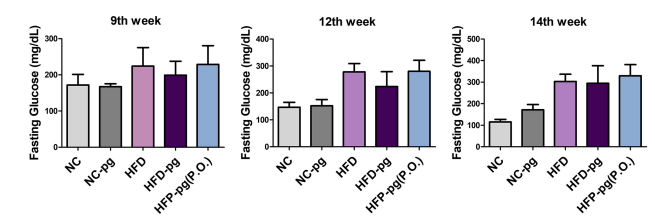

B

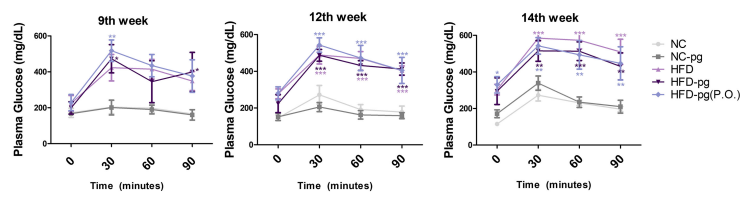

Fig. 2. Detection of blood glucose level in NC and HFD-fed mice. (A) Fasting glucose and (B) plasma glucose at 30, 60, and $90 \mathrm{~min}$ after glucose administration. The results are shown as the mean + SD. ${ }^{*} \mathrm{P}<0.05, * * \mathrm{P}<0.01, * * * \mathrm{P}<0.001$.

ing blood glucose was higher in all HFD-fed groups than in the NC group but was not altered by $P$. gingivalis administration (Fig. 2A). The IPGTTs also did not show any significant difference in glucose levels in mice infected with $P$. gingivalis via different routes. In the HFD-p.g. (P.O.) group, the glucose level in IPGTT at 9 and 12 weeks was the highest, but these changes were statistically insignificant compared to that in the HFD-fed groups (Fig. 2B). These results suggest that $P$. gingivalis treatment did not significantly aggravate HFD-induced glucose intolerance in our experimental settings.

\section{P. gingivalis exacerbates NAFLD, particularly by up-regulating lobular inflammation}

Given that fatty liver disease can be exacerbated in association with periodontitis, we then analyzed lipid accumulation, inflammation and hepatocyte degeneration in liver tissues. Upon histological examination by hematoxylin and eosin (H\&E) staining (Fig. 3A), the evaluation of steatosis, inflammation, and hepatocyte ballooning were conducted to calculate NASH score (Fig. 3B-E). Our results showed that livers of NC-p.g. group mice had minimal steatosis, and no signs of lobular inflammation and hepatocyte ballooning (NASH score 0-1). The total score of HFD-fed mice (NASH score 4-7) was significantly elevated compared to that in the NC group (Fig. 3B). The HFD-p.g. group (NASH score 5-9) exhibited significantly increased total score compared with HFD control group (NASH score 4-7). Overall, increased steatosis, lobular inflammation, and hepatocyte ballooning were displayed in the HFD groups (Fig. 3C-E). In the HFD groups infected with $P$. gingivalis (p.g. and p.g.[P.O.]), we observed extensive lobular inflammation foci (Fig. 3A). Consistently, the inflammation score was significantly increased in both $P$. gingivalis inoculated groups (Fig. 3D). This finding indicates that HFD-fed mice have steatohepatitis, which is diagnosed as a total score of 5 or higher, and $P$. gingivalis infection impairs fatty liver disease to a greater extent through the induction of increased inflammatory responses.

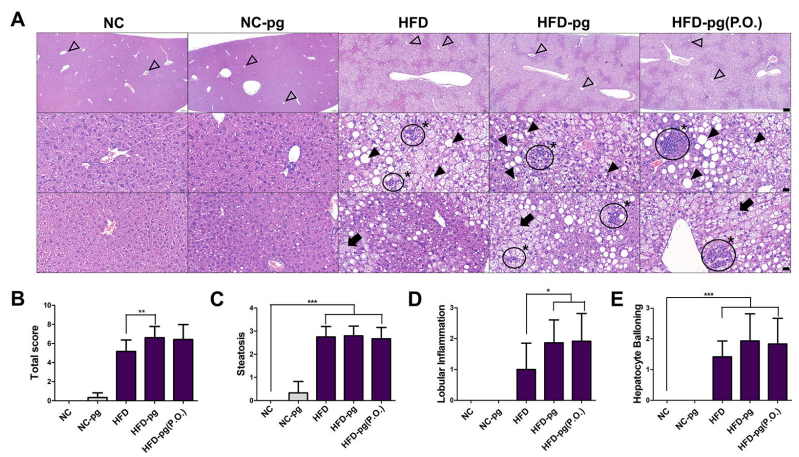

Fig. 3. Histological examination and analysis. (A) Representative H\&Estained liver tissue sections. Bar, $20 \mu \mathrm{m}$. The open arrowheads indicate the central vein and filled arrowheads indicate steatosis. The asterisks indicate lobular inflammation foci. The arrows indicate hepatocyte ballooning. (B) Total histological score, (C) steatosis, (D) lobular inflammation, and $(\mathrm{E})$ hepatocyte ballooning were exhibited. The results are shown as the mean $\pm \mathrm{SD}$. $* \mathrm{P}<0.05,{ }^{*} * \mathrm{P}<0.01$, $* * * \mathrm{P}<0.001$.

\section{$P$. gingivalis infection can exacerbate fatty liver disease through CD36 and PPAR $\gamma$ upregulation}

We next assessed the expression level of several risk factors that play a crucial role in the progression of fatty liver disease. The mRNA expression levels of genes related to inflammation and lipid or glucose metabolism in liver were determined (Fig. $4 \mathrm{~A})$. We analyzed the expression of several metabolic regulators including Foxo1, PparyC1 $\alpha, \mathrm{Cd} 36$, and Fabp4 in the liver. Decreased Foxo1 expression was observed in the HFD and HFD-p.g. groups. Importantly, the expression level of Ppary $\mathrm{C} 1 \alpha$, a key regulator of adipocyte differentiation and lipid metabolism, was significantly upregulated upon $P$. gingivalis infection in HFD-fed mice. Moreover, HFD-fed mice showed increased Cd36 mRNA expression, which increased further following $P$. gingivalis infection. The Fabp4 mRNA expression was high in HFD-fed mice, but did not show additional elevation upon $P$. gingivalis exposure. The expressions of $\operatorname{Tnf} \alpha$ and II-1 $\beta$ were upregulated in HFD-p.g., but this change was statistically insignificant. The expression levels of II- 4 and II- 17 were not altered. The mRNA levels of inflammasome components including NIrp3 and Casp1 were not changed upon HFD feeding or $P$. gingivalis infection. We next investigated the phosphorylation of Akt, AMPK and mTOR, which represent insulin responsiveness and metabolic regulation, respectively. Akt phosphorylation was slightly decreased in the HFD-fed mice group and, both intraoral and peroral administration of $P$. gingivalis did not alter the level of p-Akt (Fig. 4B). Moreover, the expression levels of p-mTOR and p-AMPK did not show any consistent pattern of change (Fig. 4C). Taken together, our findings demonstrate that $P$. gingivalis infection can exacerbate fatty liver disease by disturbing hepatic lipid metabolism through the CD36-PPAR $\gamma$ pathway. 


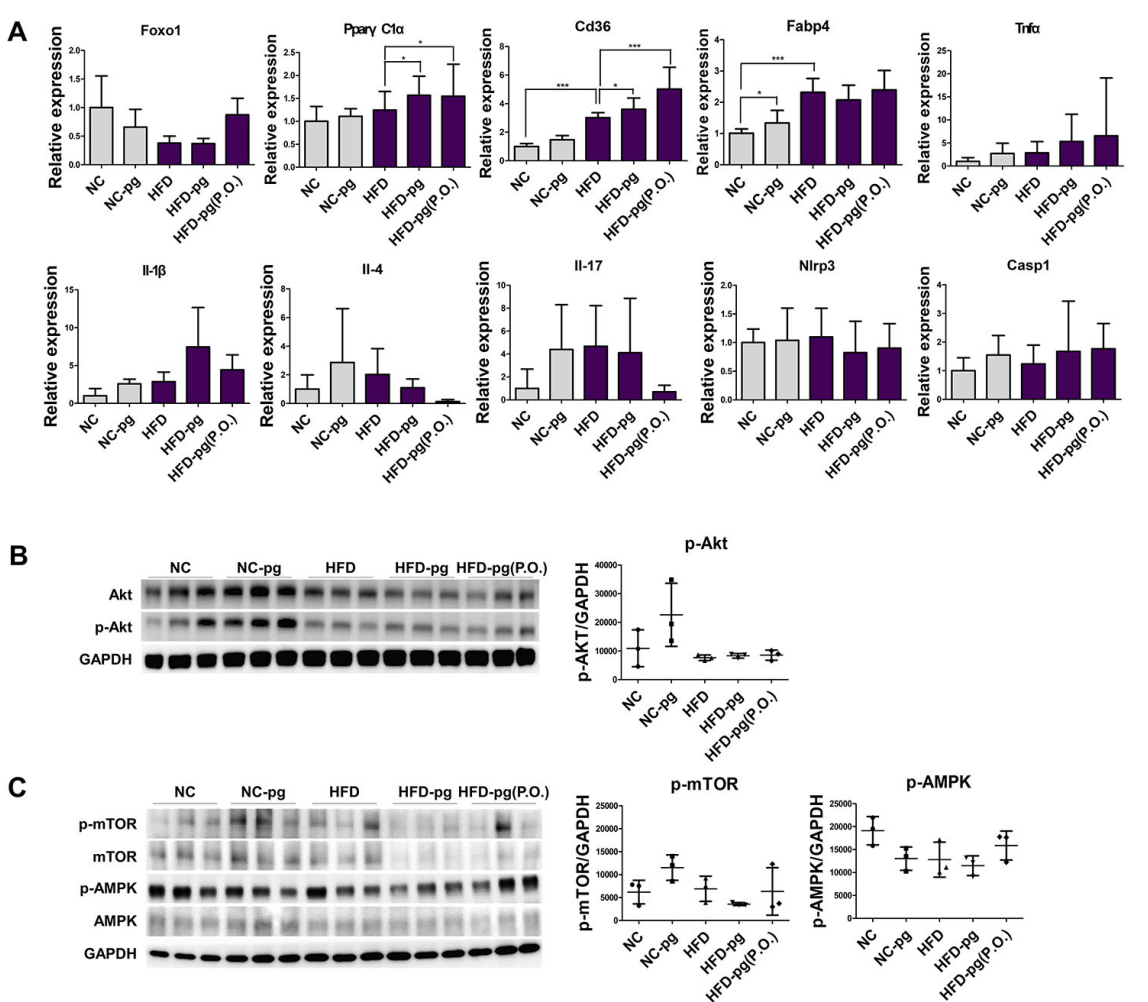

Fig. 4. The $m R N A$ and protein expression in liver tissues. (A) The mRNA expression levels of Foxo1, PparyC1 $\alpha, \mathrm{Cd} 36$ Fabp4, Tnfo, II-13, II-4, II-17, Nlrp3, and Casp1 in liver tissue. Western blot analysis of (B) Akt, p-Akt, (C) mTOR, p-mTOR, AMPK, and p-AMPK protein levels in liver tissue (3-5 mice/group). The results are shown as the mean \pm SD $* \mathrm{P}<0.05, * * \mathrm{P}<0.01, * * * \mathrm{P}<0.001$.

\section{DISCUSSION}

Periodontitis is an inflammatory disease that leads to alveolar bone loss and further exerts various adverse impacts on systemic health. It is widely accepted that immune responses to peri-odontogenic pathogen play key roles in the progression of metabolic diseases (29). In the present study, we reported that $P$. gingivalis, a major candidate correlating periodontitis and metabolic diseases (30), can aggravate fatty liver signatures in mouse models of obesity and periodontitis.

A previous study has experimentally demonstrated that $P$. gingivalis or Lipopolysaccharide (LPS) from the bacteria can impair HFD-induced insulin resistance and glucose tolerance (31). Although we used slightly modified protocols from this study, however, in our study, neither intraoral nor peroral administration of $P$. gingivalis did not significantly increase the level of fasting glucose or glucose intolerance. This discrepancy might be due to the differences in the environment of animal breeding or virulent potency in $P$. gingivalis strains. Other studies have shown consistent results with our findings. Li et al. explored the onset and severity of diabetes using mouse model of both T1D and T2D with $P$. gingivalis infection. Despite the development of alveolar bone loss, periodontitis did not alter the glucose metabolism, determined by fasting glucose, body weight change and glucose tolerance (32). In addition, another study by Wang et al. proposed the similar findings. In the

study, $P$. gingivalis inoculation did not significantly regulate the levels of fasting glucose in $\mathrm{db} / \mathrm{db}$ mice, Tallyho/Jng/ mice and streptozotocin-treated mice (33). Kuraji et al. suggested that ligature-induced periodontitis could be more effective than the infusion of peri-odontogenic bacteria without ligation (34). Further cumulative studies might be required to establish more reproducible multiple animal models for periodontitis with other systemic diseases, using a ligature-induced model with the inoculation of peri-odontogenic bacteria.

The upregulation of components of various inflammasomes in patients with periodontitis was reported by Bostanci et al. and Garcia-Hernández et al. (35). More importantly, the latter study demonstrated that NLRP3 inflammasome components were overexpressed in the gingival tissue of patients with periodontitis and uncontrolled T2D (25). Therefore, in the present study, we observed the expression of components of the inflammasome complex, including NLRP3, NLRC4, AIM2, CASP1 and IL-1 $\beta$, in both periodontal tissues and liver tissues. However, in periodontal tissues, none of the inflammasome components was significantly regulated by HFD feeding or $P$. gingivalis infection (data not shown). One can envision that $P$. gingivalis-mediated inflammatory responses in periodontal tissues might be resolved at the time point when the samples were harvested. In liver tissues, the level of IL-1 $\beta$ expression was elevated in the HFD-fed group with intraoral $P$. gingivalis infection, which may indicate inflammasome activation could 
be involved in the regulation of live metabolism.

Despite the slight symptoms in alveolar bone loss and related inflammation, we here observed that the administration of $P$. gingivalis accelerated the progression of fatty liver disease. In particular, inflammation in liver tissue was increased in HFD-fed mice with both intraoral and peroral administration of $P$. gingivalis. Previous studies revealed that an increased level of hepatic CD36 could be a pivotal regulator in the impairment of liver metabolism (36). In addition, a recent study by Ipsen et al. reported that CD36 is abnormally increased in patients with NASH and hepatic steatosis (37). Consistent with these findings, our data showed that the level of CD36 in liver tissues was increased by HFD feeding and further increased by $P$. gingivalis administration to a greater extent. Moreover, the expression of PPAR $\gamma$, a transcription factor in the downstream of CD36, was significantly upregulated in HFD-fed mice with $P$. gingivalis infection, compared to HFD-fed mice. Moreover, a large body of other previous studies have proven that PPAR $\gamma / \mathrm{CD} 36$ pathway critically contributes to HFD-induced NAFLD through the increase of free fatty acid uptake and triglyceride synthesis in the liver. These studies collectively demonstrated that the increased expression of PPAR $\gamma$ results in elevated CD36 expression, which aggravates HFD-induced fatty liver and insulin resistance $(18,38,39)$.

The limitation of our study is that the animal model we used here is not a perfect model for the study on the correlation between periodontitis and liver metabolism and that symptoms for both diseases are too mild. Therefore, further investigations using severe periodontitis model are required since damages in periodontal tissue and subsequent exacerbating effects of periodontitis on systemic disease are all cumulative. To this end, increased dosage of bacterial infection, shorter interval of bacterial inoculation, or combined treatment with molar ligation might be adopted for future study. In conclusion, our data revealed that the administration of $P$. gingivalis in HFDfed mice aggravated NASH in mice through the upregulation of CD36 and PPAR $\gamma$, pivotal regulators in lipid metabolism. These findings might propose the novel therapeutic targets in the treatment of periodontal metabolic liver diseases.

\section{MATERIALS AND METHODS}

The detailed methods are described in the "Supplementary Materials and Methods".

\section{Induction of diabetes and periodontitis mice}

8-week-old C57BI/6 wild-type (WT) mice were housed in specific pathogen-free controlled conditions on $12 \mathrm{~h}$ day light/ dark cycle. Mice were randomly divided into five groups: negative control group (NC), negative control with $P$. gingivalis treated group (NC-p.g.), high-fat diet group (HFD), high-fat diet with $P$. gingivalis treated group (HFD-p.g.) and high-fat diet with peroral administration $P$. gingivalis group (HFD-p.g.[P.O.]). For the $P$. gingivalis group, $10 \% / \mathrm{ml}$ colony-forming unit (CFU) of $P$. gingivalis suspended in $100 \mu \mathrm{l}$ PBS (Gibco, Waltham, Massachusetts, USA) with 2\% carboxy-methylcellulose (Sigma, Saint Louis, Missouri, USA) was treated at the mandibular first molars, three times a week for 6 weeks. Each group was divided into two subgroups and fed with either normal chow or high-fat diet for 14 weeks. The induction of obesity and periodontitis model was confirmed by measuring body weight every 2 weeks and measuring fasting glucose level at 9, 12 and 14 weeks. This study protocol was confirmed by Ethics Committee of Pusan National University (ethics code: PNU2019-2130). Mice were monitored for reaching criteria of humane endpoint. Mice exhibiting signs of distress, such as panting, breathlessness, abnormal bodily posture and shaggy fur, were sacrificed using $\mathrm{CO}_{2}$ asphyxiation. At the time points of tissue harvesting, mice were sacrificed by $\mathrm{CO}_{2}$ asphyxiation. Every effort was made to minimize the suffering of mice.

\section{Histological analyses}

To assess hepatic morphology and scoring, liver samples are fixed in $10 \%$ neutral buffered formalin and stained with hematoxylin and eosin. Histological scoring was performed by three-stage ranges system as follows: Degree of steatosis (0-3), lobular inflammation (0-3), hepatocyte ballooning (0-2) (40).

\section{ACKNOWLEDGEMENTS}

This work was supported by the National Research Foundation of Korea (NRF) grant funded by the Korea government (MSIT) (No. NRF-2018R1A5A2023879). We would like to thank Editage (www.editage.co.kr) for English language editing.

\section{CONFLICTS OF INTEREST}

The authors have no conflicting interests.

\section{REFERENCES}

1. Listgarten MA (1986) Pathogenesis of periodontitis. J Clin Periodontol 13, 418-430

2. Nazir MA (2017) Prevalence of periodontal disease, its association with systemic diseases and prevention. Int J Health Sci (Qassim) 11, 72-80

3. Lovegrove JM (2004) Dental plaque revisited: bacteria associated with periodontal disease. J N Z Soc Periodontol $87,7-21$

4. Kitamoto $\mathrm{S}$, Nagao-Kitamoto $\mathrm{H}$, Jiao $\mathrm{Y}$ et al (2020) The intermucosal connection between the mouth and gut in Commensal Pathobiont-Driven Colitis. Cell 182, 447-462 e414

5. Potempa J, Mydel P and Koziel J (2017) The case for periodontitis in the pathogenesis of rheumatoid arthritis. Nat Rev Rheumatol 13, 606-620

6. Seo Y, Oh SJ, Ahn JS, Shin YY, Yang JW and Kim HS (2019) Implication of Porphyromonas gingivalis in colitis and homeostasis of intestinal epithelium. Lab Anim Res 35,26 
7. de Macedo GM, Nunes S and Barreto T (2016) Skin disorders in diabetes mellitus: an epidemiology and physiopathology review. Diabetol Metab Syndr 8, 63

8. Yamazaki D, Hitomi H and Nishiyama A (2018) Hypertension with diabetes mellitus complications. Hypertens Res 41, 147-156

9. Graves DT, Ding Z and Yang Y (2020) The impact of diabetes on periodontal diseases. Periodontol 200082 , 214-224

10. Salvi GE, Carollo-Bittel B and Lang NP (2008) Effects of diabetes mellitus on periodontal and peri-implant conditions: update on associations and risks. J Clin Periodontol 35, 398-409

11. Khader YS, Dauod AS, El-Qaderi SS, Alkafajei A and Batayha WQ (2006) Periodontal status of diabetics compared with nondiabetics: a meta-analysis. J Diabetes Complications 20, 59-68

12. Hu M, Phan F, Bourron O, Ferre $P$ and Foufelle F (2017) Steatosis and NASH in type 2 diabetes. Biochimie 143, 37-41

13. Kim HJ, Lee Y, Fang S, Kim W, Kim HJ and Kim JW (2020) GPx7 ameliorates non-alcoholic steatohepatitis by regulating oxidative stress. BMB Rep 53, 317-322

14. Lee JH, Choi SB, Sung DJ et al (2020) Heterogeneity in liver histopathology is associated with GSK-3beta activity and mitochondrial dysfunction in end-stage diabetic rats on differential diets. BMB Rep 53, 100-105

15. Alazawi W, Bernabe E, Tai D et al (2017) Periodontitis is associated with significant hepatic fibrosis in patients with non-alcoholic fatty liver disease. PLoS One 12, e0185902

16. Akinkugbe AA, Slade GD, Barritt AS et al (2017) Periodontitis and non-alcoholic fatty liver disease, a populationbased cohort investigation in the Study of Health in Pomerania. J Clin Periodontol 44, 1077-1087

17. Ni J, Chen L, Zhong S et al (2018) Influence of periodontitis and scaling and root planing on insulin resistance and hepatic CD36 in obese rats. J Periodontol 89, 476-485

18. Wilson CG, Tran JL, Erion DM, Vera NB, Febbraio M and Weiss EJ (2016) Hepatocyte-specific disruption of CD36 attenuates fatty liver and improves insulin sensitivity in HFD-Fed mice. Endocrinology 157, 570-585

19. Koh JH, Shin YG, Nam SM, Lee MY, Chung CH and Shin JY (2009) Serum adipocyte fatty acid-binding protein levels are associated with nonalcoholic fatty liver disease in type 2 diabetic patients. Diabetes Care 32, 147-152

20. Kim DJ, Rho JH, Woo BH et al (2019) Periodontal pathogens modulate lipid flux via fatty acid binding protein 4. J Dent Res 98, 1511-1520

21. Rajan MR, Nyman E, Brannmark C, Olofsson CS and Stralfors P (2018) Inhibition of FOXO1 transcription factor in primary human adipocytes mimics the insulin-resistant state of type 2 diabetes. Biochem J 475, 1807-1820

22. Preshaw PM, Alba AL, Herrera D et al (2012) Periodontitis and diabetes: a two-way relationship. Diabetologia 55, 21-31

23. Cutando A, Montero J, Gomez-de Diego R, Ferrera MJ and Lopez-Valverde A (2015) Effect of topical application of melatonin on serum levels of C-reactive protein (CRP), interleukin-6 (IL-6) and tumor necrosis factor-alpha (TNFalpha) in patients with type 1 or type 2 diabetes and periodontal disease. J Clin Exp Dent 7, e628-633
24. Grover HS and Luthra S (2013) Molecular mechanisms involved in the bidirectional relationship between diabetes mellitus and periodontal disease. J Indian Soc Periodontol $17,292-301$

25. Garcia-Hernandez AL, Munoz-Saavedra AE, Gonzalez-Alva $P$ et al (2019) Upregulation of proteins of the NLRP3 inflammasome in patients with periodontitis and uncontrolled type 2 diabetes. Oral Dis 25, 596-608

26. Braunersreuther V, Viviani GL, Mach F and Montecucco F (2012) Role of cytokines and chemokines in non-alcoholic fatty liver disease. World J Gastroenterol 18, 727-735

27. Mridha AR, Wree A, Robertson AAB et al (2017) NLRP3 inflammasome blockade reduces liver inflammation and fibrosis in experimental NASH in mice. J Hepatol 66, 1037-1046

28. Shoelson SE, Herrero L and Naaz A (2007) Obesity, inflammation, and insulin resistance. Gastroenterology $132,2169-2180$

29. Zhu M and Nikolajczyk BS (2014) Immune cells link obesity-associated type 2 diabetes and periodontitis. J Dent Res 93, 346-352

30. Saito T and Shimazaki Y (2007) Metabolic disorders related to obesity and periodontal disease. Periodontol 2000 43, 254-266

31. Blasco-Baque V, Garidou L, Pomie C et al (2017) Periodontitis induced by Porphyromonas gingivalis drives periodontal microbiota dysbiosis and insulin resistance via an impaired adaptive immune response. Gut 66 872-885

32. Li H, Yang H, Ding Y et al (2013) Experimental periodontitis induced by Porphyromonas gingivalis does not alter the onset or severity of diabetes in mice. J Periodontal Res 48 , 582-590

33. Wang Q, Zhang P, Aprecio R et al (2016) Comparison of experimental diabetic periodontitis induced by Porphyromonas gingivalis in mice. J Diabetes Res 2016, 4840203

34. Kuraji $R$, Ito $H$, Fujita $M$, Ishiguro $H$, Hashimoto $S$ and Numabe $Y$ (2016) Porphyromonas gingivalis induced periodontitis exacerbates progression of non-alcoholic steatohepatitis in rats. Clin Exp Dent Res 2, 216-225

35. Bostanci N, Emingil G, Saygan B et al (2009) Expression and regulation of the NALP3 inflammasome complex in periodontal diseases. Clin Exp Immunol 157, 415-422

36. Koonen DP, Jacobs RL, Febbraio M et al (2007) Increased hepatic CD36 expression contributes to dyslipidemia associated with diet-induced obesity. Diabetes 56, 28632871

37. Ipsen DH, Lykkesfeldt J and Tveden-Nyborg P (2018) Molecular mechanisms of hepatic lipid accumulation in non-alcoholic fatty liver disease. Cell Mol Life Sci 75, 3313-3327

38. Liu Y, Cheng F, Luo Y et al (2017) PEGylated curcumin derivative attenuates hepatic steatosis via CREB/PPARgamma/CD36 pathway. Biomed Res Int 2017, 8234507

39. Zhou J, Febbraio M, Wada T et al (2008) Hepatic fatty acid transporter Cd36 is a common target of LXR, PXR, and PPARgamma in promoting steatosis. Gastroenterology $134,556-567$

40. Kleiner DE, Brunt EM, Van Natta M et al (2005) Design and validation of a histological scoring system for nonalcoholic fatty liver disease. Hepatology 41, 1313-1321 